\title{
Estimativa de biomassa vegetal e sequestro de carbono no Parque Natural Municipal Flor do Ipê, Várzea Grande, MT
}

\begin{abstract}
Estimate of biomass plant and carbon kidnapping in natural Parque Natural Municipal Flor do Ipê, Várzea Grande, MT
\end{abstract}

\section{Estimación de planta de biomasa y el secuestro carbono Parque Natural Municipal Flor do Ipê, Várzea Grande, MT}

Wilian de Oliveira Rocha ${ }^{1}$ Andressa Motta Pereira ${ }^{2}$ Anny Ediane da Silva ${ }^{2}$ Joice Aparecida Fraga ${ }^{2}$

${ }^{1}$ Engenheiro Florestal; Mestre em Ciências Florestais e Ambientais; Professor dos Cursos de Engenharia Ambiental, Agronomia e Ciências Biológicas- Área de Conhecimento em Ciências Agrárias, Biológicas e Engenharias do Centro Universitário de Várzea Grande, MT (UNIVAG). E-mail:wilianroch@hotmail.com

${ }^{2}$ Graduandas em Engenharia Ambiental pelo Centro Universitário de Várzea Grande (UNIVAG), MT. E-mail(s): andressa.motta.pereira@gmail.com; aedianesilva@gmail.com; joice_fraga@hotmail.com 
Resumo: O objetivo deste estudo foi estimar a biomassa vegetal arbórea viva da parte aérea e seu potencial de sequestro de carbono no perímetro do Parque Natural Municipal Flor do Ipê, no município de Várzea Grande, MT. A área de estudo foi amostrada a partir de cinco parcelas de $100 \mathrm{~m}^{2}$ alocadas aleatoriamente. Em cada parcela, foram inventariados os indivíduos arbóreos vivos, com DAP entre $2,5 \mathrm{~cm}$ e $30 \mathrm{~cm}$, conforme a EMBRAPA para estimativa de carbono estocado e, assim, foi indicado o potencial de sequestro de carbono. A espécie de maior dominância nas parcelas estudadas foi Astronium fraxinifolium Schott, seguida pelas espécies Zanthoxylun rhoifolium Lam. e Acrocomia aculeata (Jacq.) Lodd. Ex Mart. A estimativa total de biomassa vegetal aérea viva acumulada na área do parque foi de 460,64 ton/ha, sendo 207,29 ton/ha de carbono armazenado. O potencial estimado de sequestro de $\mathrm{CO}_{2}$ foi de 760,75 ton/ha no perímetro total do parque.

Palavras-chave: Cerrado; áreas verdes urbanas; mecanismo de desenvolvimento limpo; Ciências Ambientais.

Abstract: The aim of this study was to estimate the arboreal living biomass of shoots and their carbon sequestration potential in the perimeter of the Parque Natural Municipal Flor do Ipê, in the municipality of Várzea Grande, MT. The study area was sampled from five plots of $100 \mathrm{~m}^{2}$ randomly allocated. In each plot, they were inventoried living arboreal individuals with diameter at breast height between $2.5 \mathrm{~cm}$ and $30 \mathrm{~cm}$, according to EMBRAPA to estimate carbon stored and thus has indicated the potential for carbon sequestration. The species most dominant in plots studied was Astronium fraxinifolium Schott, followed by species Zanthoxylun rhoifolium Lam. and Acrocomia aculeata (Jacq.) Lodd. Ex Mart. The total estimated cumulative live aerial biomass in the park area was 460.64 tons/ha, 207.29 tons/ha of carbon stored. The estimated potential $\mathrm{CO}_{2}$ sequestration was 760.75 tons/ha in total perimeter of the park.

Key words: Cerrado; urban green zone; clean development mechanism; Environmental Sciences.

Resumen: El objetivo de este estudio fue estimar la biomasa arbórea habitable de brotes y su potencial de secuestro de carbono en el perímetro del Parque Natural Municipal Flor do Ipê, en el municipio de Várzea Grande, MT. El área de estudio se tomaron muestras de cinco parcelas de $100 \mathrm{~m}^{2}$ asignado al azar. En cada parcela, que fueron catalogados individuos arbóreos que vivían con DAP entre $2,5 \mathrm{~cm}$ y $30 \mathrm{~cm}$, de acuerdo con la EMBRAPA para estimar el carbono almacenado y por lo tanto nos ha indicado el potencial de captación de carbono. La especie más dominante en las parcelas estudiadas ha sido Astronium fraxinifolium Schott, seguido de especies Zanthoxylun rhoifolium Lam. y Acrocomia aculeata (Jacq.) Lodd. Ex Mart. El total estimado de biomasa aérea en vivo acumulado en el área del parque era 460.64 t/ha y 207.29 t/ha de carbono almacenado. El potencial de captura de $\mathrm{CO}_{2}$ estimada era de 760,75 t/ha en el perímetro total del parque.

Palabras clave: Cerrado; áreas verdes urbanas; mecanismo de desarrollo limpio; Ciencias Ambientales. 


\section{INTRODUÇÃO}

Atualmente, com as modificações de uso da terra e o grande avanço no desenvolvimento urbano, a vegetação e o solo têm sido substituídos por concreto e asfalto. As modificações de uso da terra aliadas ao desmatamento e à perda de biodiversidade, contribuem no aumento da emissão de gases do efeito estufa (GEE) e podem acentuar modificações climáticas na Terra.

Com a relevante preocupação mundial no que diz respeito às mudanças climáticas e à degradação do meio ambiente, em 1997, na cidade de Kyoto, durante a Terceira Conferência das Nações Unidas Sobre Mudanças Climáticas, foi elaborado o Protocolo de Kyoto, assinado por representantes de mais de 160 países, reconhecendo a necessidade de um esforço global para o enfrentamento das questões climáticas.

O Protocolo de Kyoto foi criado com o objetivo de reduzir a emissão dos gases do efeito estufa na atmosfera. A redução nas emissões que os países desenvolvidos se comprometeram a realizar foi de aproximadamente 5,2\% em relação aos níveis emitidos na década de 90, isso entre os anos de 2008 e 2012. Os países em desenvolvimento também entraram nessa proposta de redução, comprometendo-se em reduzir a emissão conforme o seu desenvolvimento. Os nomeados "mecanismos de flexibilização" foram as alternativas propostas para que sejam cumpridas essas metas de redução, que incluem a ampliação de áreas verdes em centros urbanos, aliados à minimização da supressão vegetal.

Portanto, a partir do Protocolo de Kyoto, vários estudos relacionados à emissão de gases causadores do efeito estufa, e também à captação desses gases, vêm sendo elaborados para o melhor entendimento de métodos que ajudem na redução destes na atmosfera. Uma das preocupações tem sido a conservação ou o cultivo de áreas 
arborizadas para diminuir os níveis de $\mathrm{CO}_{2}$ na atmosfera e, assim, contribuir para a saúde da população tanto quanto diminuir o aquecimento global.

Devido às grandes extensões de superfícies impermeáveis e alterações no uso dos solos, as áreas urbanas apresentam temperaturas mais altas quando comparadas com áreas menos urbanizadas. Esse efeito, denominado ilha de calor, pode acarretar temperaturas mais altas (3-5ㅇ C) que nas áreas rurais próximas.

Para ajudar a amenizar o efeito das ilhas de calor, faz-se necessária a presença de áreas verdes em meio urbano. As árvores, componente da vegetação urbana, desempenham um papel vital no bem-estar das pessoas que vivem em áreas urbanizadas, pois possuem a capacidade de controlar boa parte dos efeitos adversos do meio antrópico, melhorando assim a qualidade de vida dessas pessoas.

Essa é uma das formas mais eficientes de minimizar os impactos ligados às alterações climáticas e contribui na redução das emissões de carbono, sequestrando-o, fixando-o e mantendo-o pelo maior tempo possível na biomassa vegetal. Através da fotossíntese, as plantas utilizam o dióxido de carbono $\left(\mathrm{CO}_{2}\right)$ na síntese de carboidratos e, assim, fixam o carbono em sua biomassa.

O tema "sequestro de carbono" tem ganhado importância global por sua influência nas mudanças climáticas. Nessa problemática, faz-se necessário realizar estudos específicos de cálculos de captura de carbono pelas florestas, pois os cálculos antes realizados geralmente são baseados em dados genéricos já existentes de produção de biomassa e, nem sempre, correspondem às diferentes formações florestais existentes no Brasil.

O sequestro de carbono abrange não apenas a captura de carbono da atmosfera, mas também a prevenção de emissão de gases do efeito estufa dos ecossistemas. Assim, algumas maneiras simples, como a proteção de áreas vegetais e manejo sustentável destas, 
contribuem significativamente para o sequestro e estoque de carbono, como também para a redução de ilhas de calor.

O compartimento ambiental facilmente mensurado é a biomassa viva aérea, podendo ser utilizados métodos diretos e indiretos para essa quantificação, pois o carbono fica armazenado em diferentes compartimentos além da biomassa viva aérea (tronco, galhos e folhas), sendo encontrado também na biomassa subterrânea (raízes), na biomassa morta e na matéria orgânica do solo.

Assim, para este estudo, foi adotada uma metodologia indireta ao mensurar o carbono estocado e o consequente potencial de captação de carbono atmosférico, a partir das características diamétricas dos indivíduos arbóreos amostrados na área de estudo em meio a uma vegetação típica de matas ciliares do cerrado mato-grossense, em ambiente urbano de uma importante região metropolitana do estado.

No caso de formações savânicas como o cerrado brasileiro, é de grande relevância que estudos sobre a capacidade desde bioma, e suas diferentes fitofisionomias, em sequestrar carbono sejam realizados nos mais distintos compartimentos deste, pois essa vegetação possui características particulares influenciadas por fatores edáficos, climáticos e ocorrência de incêndios florestais, principalmente em áreas verdes urbanas caracterizadas por esse tipo de vegetação. A caracterização dessas áreas verdes urbanas, como praças e parques, é relevante devido ao fato de que elas podem acentuar o conforto térmico das cidades e contribuir com uma melhor qualidade do ar.

Diante dessa premissa, o presente estudo objetivou estimar a biomassa vegetal arbórea viva da parte aérea e seu potencial de sequestro de carbono no perímetro do Parque Natural Municipal Flor do Ipê, em Várzea Grande, MT. 


\section{MATERIAL E MÉTODOS}

\section{1 Área de estudo}

O estudo foi conduzido no Parque Natural Municipal Flor do Ipê (15 38' 32,57"S; 56 4' 56,20”W), localizado no bairro homônimo, região do Cristo Rei, no município de Várzea Grande, Mato Grosso (Figura 1). O parque possui uma área total de 4,8 ha, possuindo 750 metros de trilhas suspensas, utilizado para visitação e contemplação de fauna e flora do cerrado mato-grossense. O clima da cidade é classificado como Tropical semiúmido, com aproximadamente cinco meses secos (maio a setembro), apresentando duas estações bem definidas, uma seca (outono-inverno) e uma chuvosa (primavera-verão) (SANTOS, 2013).

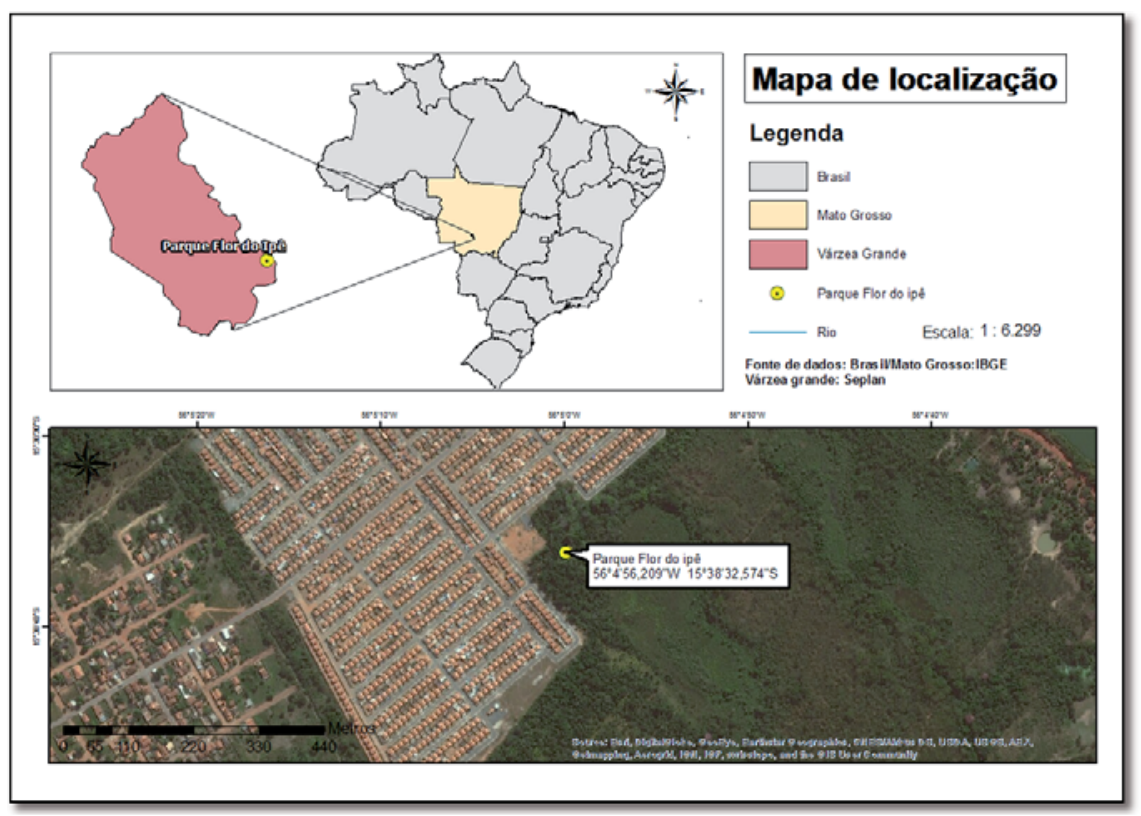

FIGURA 1 - Localização do Parque Natural Municipal Flor do Ipê no município de Várzea Grande, Mato Grosso, Brasil.

Fonte: Autoria própria. 


\subsection{Metodologia utilizada}

O estudo seguiu a metodologia desenvolvida pelo ICRAF (Centre for Research in Agroforestry), descrita e adotada por Arevalo, Alegre e Vilcahuaman (2002), publicada pela Empresa Brasileira de Pesquisa Agropecuária (EMBRAPA), para estimar o estoque de carbono em diferentes sistemas de uso da terra.

Assim, em abril de 2016, foram alocadas cinco parcelas aleatoriamente de $4 \mathrm{~m} \times 25 \mathrm{~m}$, onde foi realizado o inventário florestal, medindo o diâmetro à altura do peito (DAP) de todas as árvores que apresentaram este parâmetro entre $2,5 \mathrm{~cm}$ e $30,0 \mathrm{~cm}$, além de estimar a altura de cada indivíduo. Segundo a metodologia utilizada, quando encontrada alguma árvore com o DAP maior que $30 \mathrm{~cm}$ no interior da parcela, foi necessário ampliar o perímetro desta para $5 \mathrm{~m} \times 100 \mathrm{~m}$.

Os indivíduos mensurados no interior de cada parcela foram registrados e coletados para identificação taxonômica in loco ao nível de espécie, classificando-os com base no Angiosperm Phylogeny Group - APG III (SOUZA; LORENZI, 2012). As espécies não identificadas em campo foram levadas ao Herbário UNIVAG para possível identificação por comparação com a coleção botânica da instituição.

Nos indivíduos que apresentaram ramificações nos caules abaixo de 1,3 m a partir do nível do solo, a metodologia sugere que estime o diâmetro de ambas as ramificações e seja utilizada a seguinte equação:

\section{$\mathrm{DAP}=\mathrm{V}(\mathrm{R} 1+\mathrm{R} 2)$}

Em que:

$\mathrm{R} 1=$ ramificação 1 ;

$\mathrm{R} 2$ = ramificação 2 .

Um dos parâmetros necessários para se estimar o estoque de carbono, segundo Arevalo, Alegre e Vilcahuaman (2002), é o calculo da biomassa arbórea viva da parte aérea, que é representada por 
toda a biomassa (tronco, ramos e folhas) das árvores, sendo estimado pela equação:

\section{$B A=0,1184 D A P^{2,53}$}

Em que:

$\mathrm{BA}=$ biomassa de árvores vivas (kg/árvore);

0,1184 = constante;

$\mathrm{DAP}=$ diâmetro à altura do peito $\operatorname{DAP}(\mathrm{cm})$;

$2,53=$ constante.

Assim, foi realizada somatória da biomassa aérea de árvores vivas na parcela (BTAV), para que então, de acordo com o tamanho desta ( $4 \mathrm{~m} \times 25$ ou de $5 \mathrm{~m} \times 100 \mathrm{~m})$, fosse estimada a biomassa total de árvores vivas (BAVT):

\section{$\operatorname{BAVT}=\operatorname{BTAV}^{*} 0,1$ \\ ou \\ BAVT $=$ BTAV $* 0,02^{2}$}

Em que:

BAVT = biomassa aérea total de árvores vivas (ton/há);

BTAV = biomassa total na parcela de $4 \mathrm{~m} \times 25$ ou na de $5 \mathrm{~m} \times 100 \mathrm{~m}$;

0,01 = fator de conversão quando a parcela é de $4 \mathrm{~m} \times 25 \mathrm{~m}$;

0,02 = fator de conversão quando a parcela é de $5 \mathrm{~m} \times 100 \mathrm{~m}$.

Para a determinação do estoque de carbono, foi adotada a seguinte equação:

\section{CBAVT $=$ BAVT $* 0,45$}

Em que:

$\operatorname{CBAVT}(\mathrm{t} / \mathrm{ha})=$ carbono na biomassa aérea de árvores vivas;

BAVT = biomassa aérea total de árvores vivas (ton/ha);

$0,45=$ constante . 
Por fim, para o cálculo de carbono total da parte aérea existente de árvores vivas, foi realizada a média entre as cinco parcelas amostradas para o carbono estocado (ton/ha), e então, estimado para a área total do parque.

Para Fernandes et al. (2008), a estimativa de estoque de carbono representa a quantidade que foi removida da atmosfera e que se encontra armazenada na biomassa aérea, na forma de carbono orgânico. Para se encontrar o valor de $\mathrm{CO}_{2}$, considerou-se que uma tonelada de carbono corresponde a 3,67 toneladas de $\mathrm{CO}_{2}$. Portanto, para obter o valor de $\mathrm{CO}_{2}$ sequestrado da atmosfera, foi adotada a seguinte equação:

\section{CS (ton/ha) $=\mathrm{CTBV}^{*} 3,67$}

Em que:

CS $($ ton $/$ ha $)=$ carbono sequestrado

CTBV (ton/ha) = carbono total na biomassa aérea viva $3,67=$ constante.

\section{RESULTADOS E DISCUSSÃO}

No estudo foram inventariados 105 indivíduos arbóreos, pertencentes a 26 espécies distribuídas em 15 famílias (Tabela 1). As espécies com maior número de indivíduos presentes foram a Astronium fraxinifolium Schott, Zanthoxylun rhoifolium Lam. e Acrocomia aculeata (Jacq.) Lodd. Ex Mart. 
TABELA 1 - Famílias, espécies, nomes populares e ocorrência nas parcelas amostradas no Parque Natural Municipal Flor do Ipê, Várzea Grande, Mato Grosso, 2016

\begin{tabular}{|c|c|c|c|c|c|c|}
\hline Família/Espécie & Nome Popular & P1 & P2 & P3 & P4 & P5 \\
\hline \multicolumn{7}{|l|}{ Anacardiaceae } \\
\hline Anacardium occidentale L. & Cajueiro & & & & $x$ & $x$ \\
\hline Astronium fraxinifolium Schott. & Gonçaleiro & $\mathrm{x}$ & $x$ & $\mathrm{x}$ & $\mathrm{x}$ & $\mathrm{x}$ \\
\hline Myracrodruon urundeuva Allemão & Aroeira & & & & & $\mathrm{x}$ \\
\hline \multicolumn{7}{|l|}{ Arecaceae } \\
\hline $\begin{array}{l}\text { Acrocomia aculeata (Jacq.) Lodd. } \\
\text { Ex Mart. }\end{array}$ & Bocaiúva & $x$ & $x$ & $x$ & $x$ & \\
\hline Orbignia speciosa Mart. & Babaçú & $x$ & & & & \\
\hline \multicolumn{7}{|l|}{ Boraginaceae } \\
\hline Cordia ecalyculata Vell. & Chá-de-frade & & & $\mathrm{x}$ & $x$ & \\
\hline \multicolumn{7}{|l|}{ Fabaceae } \\
\hline $\begin{array}{l}\text { Anadenanthera falcata (Benth.) } \\
\text { Speg. }\end{array}$ & $\begin{array}{l}\text { Angico-branco/ } \\
\text { Angico-liso }\end{array}$ & & & & $x$ & \\
\hline $\begin{array}{l}\text { Anadenanthera colubrina (Vell. } \\
\text { Brenan) }\end{array}$ & Angico-de-espinho & & & & & $x$ \\
\hline Bauhinia rufa (Bong.) Steud. & Pata-de-vaca & & $x$ & & & \\
\hline Dalbergia miscolobium Benth. & $\begin{array}{l}\text { Jacarandá- } \\
\text {-do-cerrado }\end{array}$ & $\mathrm{x}$ & & & & \\
\hline Erythrina fusca Lour. & Abobreiro & & & $\mathrm{x}$ & & \\
\hline $\begin{array}{l}\text { Senegalia polyphylla (DC.) Britton } \\
\text { \& Rose }\end{array}$ & Espinheiro & $x$ & & & & $x$ \\
\hline \multicolumn{7}{|l|}{ Lamiaceae } \\
\hline Vitex cymosa Bert. & $\begin{array}{c}\text { Pimenta-de-arancuã/ } \\
\text { Tarumã }\end{array}$ & $x$ & & & $x$ & \\
\hline \multicolumn{7}{|l|}{ Lythraceae } \\
\hline Lafoensia pacari A. St.-Hil. & Pau-de-bicho & $x$ & & & $x$ & \\
\hline \multicolumn{7}{|l|}{ Malvaceae } \\
\hline Guazuma ulmifolia Lam. & Chico-magro & $x$ & & & & \\
\hline Luehea divaricata Mart. \& Zucc. & Açoita-cavalo & & & & $\mathrm{x}$ & \\
\hline Sterculia striata St.-Hil et Naud. & Manduví & $x$ & & & & \\
\hline \multicolumn{7}{|l|}{ Primulaceae } \\
\hline $\begin{array}{l}\text { Rapanea ferruginea (Ruiz e Pav.) } \\
\text { Mez }\end{array}$ & Pururuca & & $x$ & & & \\
\hline
\end{tabular}




\begin{tabular}{|c|c|c|c|c|c|}
\hline Família/Espécie & Nome Popular & P1 P2 & $2 \quad \mathrm{P3}$ & 3 P4 & P5 \\
\hline
\end{tabular}

Agonandra brasiliensis Miers ex Benth. \& Hook.

Cerveja-de-pobre $\quad \mathrm{x}$

\begin{tabular}{|c|c|c|c|c|c|}
\hline Proteaceae & & & & & \\
\hline Roupala brasiliensis Klotzsch & Carne-de-vaca & & & & $x$ \\
\hline Rhamnaceae & & & & & \\
\hline Rhamnidium elaeocarpum Reissek & Cabriteiro & & $\mathrm{x}$ & $x$ & \\
\hline Rutaceae & & & & & \\
\hline Zanthoxylun rhoifolium Lam. & Mamica-de-porca & & & $x$ & \\
\hline Salicaceae & & & & & \\
\hline Casearia gossypiosperma Briq. & Espeto & & $x$ & & \\
\hline Sapindaceae & & & & & \\
\hline Dilodendron bipinnatum Radlk. & Maria-pobre & $\mathrm{x}$ & $x$ & & $x$ \\
\hline Simaroubaceae & & & & & \\
\hline Simarouba versicolor A. St.-Hil. & Pau-de-Perdiz & & & & $x$ \\
\hline N.I & Barreiros & $\mathrm{x}$ & & & \\
\hline N.I & Fruto-de-boi & & & $x$ & \\
\hline N.I & Tambuatá & & & $x$ & \\
\hline N.I & N.I & $x$ & & & $x$ \\
\hline
\end{tabular}

Legenda: P1: Parcela 01; P2: Parcela 02; P3: Parcela 03; P4: Parcela 04; P5: Parcela 05; N.I.: Não identificada.

Os valores de biomassa e carbono variaram de acordo com as espécies encontradas, tamanho e localização da área de amostra.

Para a comparação, foram considerados os valores de carbono estocado de forma individual, ou seja, por indivíduo, pois, em alguns casos, o resultado da soma de todas as árvores de uma mesma espécie, eram inferiores ao valor encontrado para uma única árvore de outra espécie.

Ao iniciar o estudo, na primeira parcela foram amostradas árvores com diâmetros maiores de $30 \mathrm{~cm}$ que, para a metodologia utilizada, é um fator relevante, e obriga o aumento da parcela. Portanto foi obtida uma amostragem de 36 indivíduos arbóreos, e as espécies que predominaram destes foram Dilodendron bipinnatum e Astronium 
fraxinifolium. As espécies que mais estocaram carbono individualmente foram a Dilodendron bipinnatum (1,19 ton/ha) e Orbignia speciosa (1,16 ton/ha) que, apesar de ambas possuírem apenas uma unidade dentro da parcela, contribuíram de forma significativa no sequestro de carbono devido ao porte do caule e altura destas. As espécies que possuíram indivíduos com menor estoque de carbono foram a Astronium fraxinifolium (0,0001 ton/ha) e Guazuma ulmifolia (0,0007 ton/ha), respectivamente.

A partir da segunda parcela, não foram encontradas árvores com diâmetros maiores que $30 \mathrm{~cm}$, portanto as parcelas amostrais continuaram com área de $4 \mathrm{~m} \times 25 \mathrm{~m}$. Assim, na parcela 2 foram amostrados 18 indivíduos, sendo Rapanea ferruginea e Astronium fraxinifolium as espécies com maior quantidade de indivíduos. Porém as espécies que mais estocaram carbono individualmente foram Astronium fraxinifolium (0,13 ton/ha) e Acrocomia aculeata (0,05 ton/ha), respectivamente. Apesar de ser a espécie com maior estoque de carbono nesta parcela, a Astronium fraxinifolium (0,0003 ton/ha) também possuiu indivíduos com baixo estoque de carbono, em seguida, a Rapanea ferruginea (0,0005 ton/ha).

Já na terceira parcela, foram encontrados 24 espécimes, em que se observou a dominância de Astronium fraxinifolium. Nessa área, foi analisado que as árvores estão em desenvolvimento (menos desenvolvidas) e, portanto, não há ainda uma grande quantidade de carbono estocado. No entanto as espécies que mais estocaram carbono individualmente foram Acrocomia aculeata (0,05 ton/ha) e Astronium fraxinifolium (0,09 ton/ha). As espécies com menor estoque de carbono foram a Zanthoxylun rhoifolium e fruto-de-boi (família e espécie não identificadas) ambas com estoque de 0,0001 ton/ha.

Na quarta parcela, foram encontrados 20 indivíduos, na qual foi observada predominância de Anadenanthera falcata e Anacardium occidentale. Como na parcela anterior, nesta área, foi observado que 
as árvores estão em desenvolvimento e, portanto, não há ainda uma grande quantidade de carbono estocado. Ainda assim, as espécies que mais estocaram carbono individualmente foram Astronium fraxinifolium (0,12 ton/ha), Acrocomia aculeata (0,07 ton/ha) e Anacardium occidentale (0,05 ton/ha). As espécies com menor estoque de carbono foram Anadenanthera falcata (0,0005 ton/ha) e Luehea divaricata (0,0006 ton/ha), respectivamente.

Na quinta e última parcela, foram amostrados apenas sete indivíduos arbóreos, sendo aquela que possuiu a menor quantidade de árvores com mais de 2,5cm de diâmetro e que se enquadravam no estudo. Essa variação na quantidade de indivíduos pode estar relacionada à localização da parcela dentro do parque, pois é próximo à área de bordadura do parque e intermitente alagamento. Áreas de borda estão mais susceptíveis a pressões externas, apresentando um menor desenvolvimento florestal. Nesta última amostra foi encontrada apenas uma árvore de cada espécie, e as que mais estocaram carbono individualmente foram Senegalia polyphylla (0,28 ton/ ha) e Myracrodruon urundeuva (0,05 ton/ha), respectivamente. As espécies com menor estoque de carbono para esta parcela foram a Anacardium occidentale (0,0005 ton/ha) e Anadenanthera colubrina (0,0009 ton/ha).

Em seguida, a realização dos cálculos com as amostras levantadas em campo apresentou, para a primeira parcela, a quantidade de 156,98 ton/ha de biomassa vegetal, sendo 70,64 ton/ha de carbono estocado na biomassa e 12,96 ton/ha de $\mathrm{CO}_{2}$ sequestrado. Para a segunda parcela, foi encontrado 75,58 ton/ha de biomassa vegetal, sendo 34,01 ton/ha de carbono estocado e 1,25 ton/ha de $\mathrm{CO}_{2}$ sequestrado. Já na terceira parcela amostral, foi calculado 59,85 ton/ ha de biomassa vegetal, 26,93 ton/ha carbono estocado nesta e 0,99 ton/ha de $\mathrm{CO}_{2}$ sequestrado. Na quarta parcela, ocorreu 109,74 ton/ha de biomassa vegetal, 49,38 ton/ha carbono estocado e 1,81 ton/ha 
de $\mathrm{CO}_{2}$ sequestrado. Por fim, a quinta apresentou 77,69 ton/ha de biomassa vegetal, 34,96 ton/ha carbono estocado e 1,28 ton/ha de $\mathrm{CO}_{2}$ sequestrado. A média obtida no estudo para biomassa vegetal foi de 37,85 ton/ha e 17,03 ton/ha para carbono estocado e 62,514 ton/ha para $\mathrm{CO}_{2}$ sequestrado.

Em estudo de uma área do pantanal, na Nhecolândia, Mato Grosso do Sul, Fernandes et al. (2008) encontraram em uma área de 0,25 ha, a biomassa estimada de 58,695 ton/ha, sendo 29,348 ton/ha de carbono estocado e 107,707 ton/ha de $\mathrm{CO}_{2}$ sequestrado, enquanto que, para o cerrado da região de Pará de Minas, Minas Gerais, no estudo realizado por Lopes e Miola (2010), estes encontraram numa área de 0,1 ha, a biomassa estimada de 7,33 ton/ha, 3,30 ton/ha de carbono e 12,11 ton/ha de $\mathrm{CO}_{2}$. Observa-se que há uma discrepância de valores e que está relacionada às diferentes metodologias utilizadas, diferenças na dimensão territorial e na pedologia, os diferentes tipos de espécies, além do estágio sucessional da vegetação das áreas estudadas e, consequente, ciclagem de nutrientes.

A Tabela 2 apresenta os valores de carbono estocado na biomassa, em diferentes áreas de Cerrado no Brasil, estimados para uma área de um hectare, demonstrando comparativamente, as diferenças com este estudo e, ainda, a grande variação que pode ocorrer nesse bioma em relação à biomassa vegetal estocada, o que justifica novas e intensificadas pesquisas nessa temática ao longo das diferentes fitofisionomias savânicas brasileiras.

Tabela 2 - Estoque de carbono em biomassa arbórea viva em diferentes regiões de Cerrado no Brasil

\begin{tabular}{cccc} 
& \multicolumn{3}{c}{ Valores médios de carbono estocados em ton/ha } \\
\cline { 2 - 4 } Fitofisionomia & $\begin{array}{c}\text { Fernandes et al. } \\
(2008)\end{array}$ & $\begin{array}{c}\text { Lopes e Miola } \\
(2010)\end{array}$ & $\begin{array}{c}\text { Pessoa e Martins } \\
(2015)\end{array}$ \\
\hline Cerrado & 117,392 & 33 & 62,070 \\
\hline
\end{tabular}

Fonte: Autores citados. 
Pessoa e Martins (2015), em seu estudo de sequestro de carbono no município de Dom Aquino, obtiveram o valor de 277,8 ton/ha, que, ao comparar com a área total do Parque Flor do Ipê, estima-se aproximadamente 1333,44 ton/ha. O conhecimento desse carbono armazenado pode contribuir economicamente para municípios brasileiros, gerando rentabilidade através da comercialização de créditos de carbono, além de programas consolidados de planejamento e economia ambiental dentro dessas cidades (VIDIGAL, s.d.).

É necessário ressaltar a complexidade quanto à comparação de dados que envolvam ao sequestro de $\mathrm{CO}_{2}$, devido às diferentes metodologias e as variadas dimensões de áreas amostradas e o estágio sucessional da vegetação. Essas metodologias distintas variam, também, de acordo com o compartimento estocado analisado, pois o carbono fica armazenado em diferentes órgãos vegetais, como a biomassa viva aérea (tronco, galhos e folhas) empregada neste estudo, possibilitando ser estimado na biomassa subterrânea (raízes), na biomassa morta e na matéria orgânica do solo (LAERA, 2006; FERNANDES et al., 2008; SILVA et al., 2014).

O manejo de áreas arborizadas em ambientes urbanos tendem a diminuir os níveis de $\mathrm{CO}_{2}$ na atmosfera, auxiliando a saúde da população e minimizando o aquecimento global, porém observam-se na literatura poucos estudos desse tipo estritamente para áreas verdes urbanas no estado de Mato Grosso, quando comparados às demais regiões do Brasil (MALAVASI; MALAVASI, 2001; PANDEY, 2002; GOMES; SOARES, 2003; LOPES; MIOLA, 2010; SANTOS, 2013; SILVA et al., 2014).

\section{CONCLUSÃO}

Após análises, concluiu-se que a estimativa total de biomassa aérea vegetal viva acumulada é de 460,64 toneladas em toda área do parque e 207,29 toneladas de carbono armazenado, sendo que esse 
compartimento da vegetação analisada sequestrou 760,75 toneladas de $\mathrm{CO}_{2}$ no perímetro do parque até então.

Recomenda-se que outros compartimentos da vegetação (arbustivo/herbáceo, árvores mortas caídas e/ou ainda eretas, serapiIheira, entre outros) sejam abordados em estudos posteriores nesse parque para que, assim, obtenha-se uma estimativa completa do $\mathrm{CO}_{2}$ sequestrado por essa área verde no município de Várzea Grande, Mato Grosso.

\section{Agradecimentos}

À Secretaria de Meio Ambiente e Desenvolvimento Rural Sustentável de Várzea Grande e à Prefeitura Municipal de Várzea Grande, estado de Mato Grosso, Brasil.

\section{REFERÊNCIAS}

AREVALO, L. A.; ALEGRE, J. C.; VILCAHUAMAN, L. J. M. Metodologia para estimar o estoque de carbono em diferentes sistemas de uso da terra. Colombo, PR: Embrapa Floresta, 2002. 38 p. (Documentos 73).

FERNANDES, A.; SALIS, S. M.; FERNANDES, F.; CRISPIM, S. Estoques de carbono do estrato arbóreo de cerrados no Pantanal da Nhecolândia. Corumbá, MS: Embrapa Pantanal, 2008. (Comunicado Técnico 68).

GOMES, M. A. S.; SOARES, B. R. A vegetação nos centros urbanos: considerações sobre os espaços verdes em cidades médias brasileiras. Estudos Geográficos, Rio Claro, SP, v. 1, n. 1, p. 19-29, jun. 2003. Disponível em: $<$ http://www.cchla.ufrn.br/geoesp/arquivos/artigos/ArtigoAmbientePracas. pdf>. Acesso em: 2 fev. 2016.

LAERA, H. N. L. Valoração econômica da arborização: a valoração dos serviços ambientais para a eficiência e manutenção do recurso ambiental urbano. 2006. 132 f. Dissertação (Pós-Graduação em Ciência Ambiental) Universidade Federal Fluminense, Niterói, RJ, 2006. Disponível em: <http:// www.uff.br/cienciaambiental/dissertacoes/LHNLaera.pdf>. Acesso em: 3 out. 2015. 
LOPES, R. B.; MIOLA, D. T. B. Sequestro de carbono em diferentes fitofisionomias do cerrado. SynThesis Revista Digital FAPAM, Pará de Minas, MG, v. 2, n. 2, p. 127-143, nov. 2010. Disponível em: <http://fapam.web797. kinghost.net/revista/volume2/L\%20Rosimeire\%20127-143.PDF>. Acesso em: 10 jun. 2015.

MALAVASI, U. C.; MALAVASI, M. M. Avaliação da arborização urbana pelos residentes - estudo de caso em Mal. Cândido Rondon, Paraná. Ciência Florestal, Santa Maria, RS, v. 11, n. 1, p. 189-193, 2001.

PANDEY, D. N. Global climate change and carbon management in multifunctional forests. Current Science, Bengaluru, v. 83, n. 5, p. 593-602, set. 2002. Disponível em: <http://www.iisc.ernet.in/currsci/sep102002/593. pdf>. Acesso em: 10 jun. 2015.

PESSOA, S. G.; MARTINS, M. A. Sequestro de carbono em região de cerrado em mato grosso: contribuição para o equilíbrio do clima. Connection line, Várzea grande, n. 12, 2015. Disponível em: <http://www.periodicos.univag.com.br/ index.php/CONNECTIONLINE/article/view/213>. Acesso em: 7 jun. 2016.

SANTOS, F. M. M. Clima urbano de Cuiabá-MT-Brasil: ocupação do solo e suas influências. Revista Monografias Ambientais - REMOA, Santa Maria, RS, v. 12, n. 12, p. 2749-2763, ago. 2013. Disponível em: <http://webcache. googleusercontent.com/search?q=cache:http://periodicos.ufsm.br/remoa/ article/download/9160/pdf\&gws_rd=cr\&ei=k-tyV9v0Ncm2wATjgJ-4BQ>. Acesso em: 28 jun. 2016.

SILVA, A. C. S.; CAIONI, C.; VEIGA, J. B.; NETO, R. M. R.; OLIVEIRA, A. S. Estoque de biomassa aérea, carbono e sequestro de dióxido de carbono em sistemas florestais na Amazônia Mato-grossense. Revista Acta Iguazu, Cascavel, PR, v. 3, n. 4, p. 22-35, 2014. Disponível em: <http://e-revista.unioeste.br/index. php/actaiguazu/article/view/11243>. Acesso em: 11 jun. 2016.

SOUZA, V. C.; LORENZI, H. Botânica sistemática: guia ilustrado para identificação das familias de Fanerógamas nativas e exóticas no Brasil, baseado em APG III. 3. ed. Nova Odessa, SP: Instituto Plantarum de Estudos da Flora, 2012.

VIDIGAL, F. A. M. O protocolo de Kyoto, o mecanismo de desenvolvimento limpo e as formas de circulação dos créditos de carbono. [s.d.]. Disponível em: <http://www.domtotal.com/direito/uploads/11.pdf>. Acesso em: 10 jun. 2016. 
